BULL. AUSTRAL. MATH. SOC.

VOL. I5 (1976), I11-116.

\title{
A fixed point theorem for a family of nonexpansive mappings
}

\section{V.M. Sehgal}

Let $E$ be a separated, locally convex topological vector space and $F$ a commutative family of nonexpansive mappings defined on a quasi-complete convex (not necessarily bounded) subset $X$ of $E$. In this paper, it is proved that if one of the mappings in $F$ is condensing with a bounded range then the family $F$ has a common fixed point in $X$. This result improves several wellknown results and supplements a recent result of $E$. Tarafdar (Bul2. Austral. Math. Soc. 13 (1975), 241-254) for such mappings.

\section{Introduction}

Let $E$ be a separated, locally convex topological vector space and $U$ a neighborhood basis of the origin consisting of absolutely convex subsets of $E$. For each $U \in U$, let $p_{U}$ be the Minkoswki's functional of $U$. Let $P=\left\{p_{U}: U \in U\right\}$. Let $X$ be a nonempty subset of $E$. A mapping $f: X \rightarrow X$ is called $P$-nonexpansive (see Tarafdar [7]) if for each $p \in P$ and for all $x, y \in X, p(f(x)-f(y)) \leq p(x-y)$. In a recent paper [7], Tarafdar considered a commutative family of such nonexpansive mappings and proved the following extension of an earlier result of Belluce and Kirk [2].

THEOREM 1. Let $E$ be quasi-complete and $X$ a nonempty, bounded, closed, and convex subset of $E$ and $M$ a compact subset of $X$. If $F$ is a commutative family of $P$-nonexpansive mappings on $X$ having the property: there exists a $g \in F$ such that for each $x \in X$,

Received 5 April 1976. 


$$
\operatorname{cl}\left\{g^{n}(x): n \in I \text { (positive integers) }\right\} \cap M \neq \emptyset,
$$

then the fomily $F$ has a common fixed point in $M$.

In the present paper, we prove a common fixed point theorem for the family $F$ in Theorem 1 , when $X$ therein is not necessarily bounded and instead of ( 1 ) we assume the existence of a $g \in F$ which is condensing with a bounded range. The main result of this paper generalizes a wellknown result of DeMarr [3] and extends to locally convex spaces a result of Bakhtin [1], supplementing a recent result of Tarafdar [7] for such a family $F$.

\section{1.}

Recall that a closed subset $X$ of $E$ is called quasi-complete if its closed and bounded subsets are complete. Furthermore, a subset $T$ of $E$ is totally bounded if for each $U \in U$, there exists a finite subset $F$ of $T$ such that $T \subseteq E+U=\{a+b: a \in F, b \in U\}$. For a subset $S$ of $E$, let

$$
Q(S)=\{U \in U: S \subseteq T+U \text {, for some totally bounded subset } T \text { of } E\} \text {. }
$$

Let $X$ be a nonempty subset of $E$. Following Su and Sehgal [6] (see also Himmelberg, Porter and Van Vleck [4]) a mapping $f: X \rightarrow E$ is called condensing if for each bounded but not totally bounded subset $A \subseteq X$, $Q(A) \underset{f}{\subsetneq} Q(f(A))$. Note, that if $A$ is a totally bounded subset of a quasicomplete subset $X$ of $E$, then the closure of $A(c l A)$ is compact.

\section{2.}

The following result is basic to the main result of this paper.

THEOREM 2. Let $X$ be a non-empty quasi-complete convex subset of $E$ and $g: X \rightarrow X$ be a continuous condensing mapping such that $g(X)$ is a bounded subset of $X$. Then $G=\{x \in X: g(x)=x\}$ is a nonempty compact subset of $X$.

Proof. That the set $G$ is non-empty is a consequence of a result of Su and Sehgal ([6], Lemma 1 ). Furthermore, the continuity of $g$ implies that $G$ is closed and being bounded, it follows that $G$ is complete. Since

$$
Q\{(G))=Q(G)
$$


and $g$ is condensing, therefore $G$ is totally bounded and hence a compact subset of $X$.

In [7] (Lemma 2.2), Tarafdar has proved the following results.

LEMMA 1. Let $M$ be a compact subset of $E$. If for some $p \in P$,

$$
d_{p}=\sup \{p(x-y): x, y \in M\}>0 \text {, }
$$

then there is a $u$ in the convex hull of $M(\operatorname{co}(M))$ such that

$$
r=\sup \{p(x-u): x \in M\}<d_{p} .
$$

The following is the main result of this paper and is related to the lines of argument in [1].

THEOREM 3. Let $X$ be a nonempty, quasi-complete convex subset of $E$ and $F$ a commutative family of $P$-nonexpansive self mappings of $X$ satisfying the condition:

(4) there exists a $g \in F$ such that $g$ is condensing and $g(x)$ is bounded.

Then the farily $F$ has a common fixed point in $X$.

Proof. Let

$A=\{S \subseteq X: S$ is nonempty, convex, and $f(S) \subseteq S$ for each $f \in F\}$. Clearly $X \in \mathrm{A}$. Define a partial order $<$ in $\mathrm{A}$ by $S_{1}<S_{2}$ iff $S_{2} \subseteq S_{1}$. We show that any chain in $\mathrm{A}$ has an upper bound. Let $\left\{S_{\alpha}: \alpha \in \Delta\right\}$ be a chain in $A$. Let $A=\cap\left\{S_{\alpha}: \alpha \in \Delta\right\}$. Since a $P$-nonexpansive mapping is continuous, it follows by Theorem 2 that, for each $\alpha \in \Delta$,

$$
F_{\alpha}=\left\{x \in S_{\alpha}: g(x)=x\right\}
$$

is a nonempty compact subset of $S_{\alpha}$. Thus $F=\cap\left\{F_{\alpha}: \alpha \in \Delta\right\}$ is a nonempty subset of $A$. It is clear now that $A \in A$ and that $A$ is an upper bound of the chain $\left\{S_{\alpha}: \alpha \in \Delta\right\}$. Therefore, by Zorn's Lemma, there exists a minimal nonempty convex set $S_{0} \subseteq X$ such that $f\left(S_{0}\right) \subseteq S_{0}$ for each $f \in F$. Let 


$$
F=\left\{x \in S_{0}: g(x)=x\right\}
$$

Then $F$ is a nonempty compact subset of $S_{0}$ and since for any $f \in F$ and $x \in F$,

$$
f(x)=f(g(x))=g(f(x)),
$$

it follows that $f(F) \subseteq F$ for each $f \in F$. Let

$$
B=\{C \subseteq X: C \text { is nonempty, compact, and } f(C) \subseteq C \text { for each } f \in F\} \text {. }
$$

Then $F \in B$. Define the same partial order in $B$ as in $A$. Then it is easy to show by Zorn's Lemma that there is a minimal nonempty compact set $M \subseteq X$ such that $f(M) \subseteq M$ for each $f \in F$. Clearly

$$
M \subseteq S_{0}
$$

and the minimality of $M$ in $B$ implies that

$$
f(M) \equiv M \text { for each } f \in F \text {. }
$$

We show that $M$ consists of exactly one element of $X$. Suppose not. Then, since $E$ is separated, there is a $p \in P$ satisfying (2), and hence by Lemma 1 , there is a $u \in c o(M)$ satisfying (3). Now, $S_{0}$ being convex, it follows by (5) that $u \in S_{0}$. Let for each $x \in M$ and $r$ given by $(3)$,

$$
V(x)=\{z \in E: p(x-z) \leq r\} .
$$

Then $V(x)$ is convex and $u \in V(x)$ for each $x \in M$. Set

$$
V=\{V(x): x \in M\} \text { and } S=S_{0} \cap V \text {. }
$$

Clearly $S$ is convex and $u \in S$. We show that $f(S) \subseteq S$ for each $f \in F$. Since $f\left(S_{0}\right) \subseteq S_{0}$, it suffices to show that $f(V) \subseteq V$ for each $f \in F$. Let $z \in V$ and $f \in F$. Then, by (7),

$$
p(x-z) \leq r
$$

for each $x \in M$. Now for each $x \in M$, it follows by (6) that there is a $y=y(x) \in M$ such that $f(y)=x$ and hence, by (8),

$$
p(f(z)-x)=p(f(z)-f(y)) \leq p(z-y) \leq r
$$

for each $x \in M$. Thus $f(z) \in V(x)$ for each $x \in M$; that is $f(S) \subseteq S$ 
for each $f \in F$. Thus $S \in A$, and by (7) and the minimality of $S_{0}$ in A ,

$$
S=s_{0}
$$

Now $p$ being continuous and $M$ compact, there are elements $x$ and $y$ in $M$ such that $p(x-y)=d_{p}$. This equality implies that $y \notin V(x)$ and consequently $y \notin S$. However, by (5), $y \in S_{0}$. This contradicts (8). Thus $M=\{x\}$ for some $x \in X$ and hence $f(x)=x$ for each $f \in F$. This completes the proof of Theorem 3.

If $E$ is a Banach space and $u_{0}$ is the collection of spherical neighborhoods of the origin with $P_{0}=\left\{P_{U}: U \in U_{0}\right\}$, then a mapping $f$ on a subset $X$ of $E$ is $P_{0}$-nonexpansive iff $f$ is nonexpansive; that is $\|f(x)-f(y)\| \leq\|x-y\|$ for all $x, y \in X$. Further, if $f: X \rightarrow E$ is condensing in the sense of Sadovskir [5], then $f$ is condensing with respect to $u_{0}$ (see [4]). Therefore, as a consequence of Theorem 3, we have the following extension of a result of Bakhtin [2].

COROLLARY 1. Let $X$ be a nonempty, closed and convex subset of a Banach space $E$ and $F$ a commtative family of nonexpansive self mappings of $X$ satisfying the condition: there exists a $g \in F$ such that $g$ is condensing in the sense [5] with $g(X)$ bounded. Then the family $F$ has $a$ common fixed point in $X$.

COROLLARY 2. Let $X$ be a nonempty, closed, and convex subset of a Banach space $E$ and $F$ a commutative family of nonexpansive self mappings of $X$. If for some $g \in F, g(X)$ is contained in a compact subset of $X$, then the family $F$ has a common fixed point in $X$.

It may be remarked that Corollary 2 contains a result of DeMarr [3].

\section{References}

[1] I.A. Bakhtin, "Existence of common fixed points for commutative sets of nonlinear operators", Functional Anal. App Z. 4 (1970), 76-77. 
[2] L.P. Belluce and W.A. Kirk, "Fixed-point theorems for families of contraction mappings", Pacific J. Math. 18 (1966), 213-217.

[3] Ralph DeMarr, "Comon fixed points for commuting contraction mappings", Pacific J. Math. 13 (1963), 1139-1141.

[4] C.J. Himmelberg, J.R. Porter and F.S. Van Vleck, "Fixed point theorems for condensing multifunctions", Proc. Amer. Math. Soc. 23 (1969), $635-641$.

[5] B.N. Sadovskiī, "On a fixed point principle", Functional Anal. Appl. 1 (1967), 151-153.

[6] C.H. Su and V.M. Sehgal, "Some fixed point theorems for condensing multifunctions in locally convex spaces", Proc. Amer. Math. Soc. 50 (1975), 150-154.

[7] E. Tarafdar, "Some fixed-point theorems on locally convex linear topological spaces", Bull. Austral. Math. Soc. 13 (1975), 241-254.

Department of Mathematics,

University of Wyoming,

Laramie,

Wyoming,

USA. 